Luis Antonio Castro-Castro; Darwin Gabriel García-Herrera; Carlos Marcelo Ávila-Mediavilla; Juan Carlos Erazo-Álvarez

http://dx.doi.org/10.35381/r.k.v5i5.1038

\title{
Impacto de COVID-19 en Educación básica y bachillerato: Unidades educativas urbanas de Suscal - Ecuador
}

\author{
Impact of COVID-19 on Basic Education and High School: Suscal Urban \\ Educational Units - Ecuador
}

\author{
Luis Antonio Castro-Castro \\ luisantonio.castro@psg.ucacue.edu.ec \\ Universidad Católica de Cuenca, Azogues \\ Ecuador \\ https://orcid.org/0000-0002-5021-167X \\ Darwin Gabriel García-Herrera \\ dggarciah@ucacue.edu.ec \\ Universidad Católica de Cuenca, Azogues \\ Ecuador \\ https://orcid.org/0000-0001-6813-8100 \\ Carlos Marcelo Ávila-Mediavilla \\ cavilam@ucacue.edu.ec \\ Universidad Católica de Cuenca, Cuenca \\ Ecuador \\ https://orcid.org/0000-0002-2649-9634 \\ Juan Carlos Erazo-Álvarez \\ jcerazo@ucacue.edu.ec \\ Universidad Católica de Cuenca, Cuenca \\ Ecuador \\ https://orcid.org/0000-0001-6480-2270
}

Recibido: 15 de septiembre de 2020

Revisado: 19 de octubre de 2020

Aprobado: 15 de noviembre de 2020

Publicado: 01 de diciembre de 2020 
Luis Antonio Castro-Castro; Darwin Gabriel García-Herrera; Carlos Marcelo Ávila-Mediavilla; Juan Carlos Erazo-Álvarez

\title{
RESUMEN
}

Se tuvo como objetivo analizar el impacto de COVID-19 en Educación básica y bachillerato: Unidades educativas urbanas de Suscal - Ecuador. Fue realizada en las instituciones educativas ubicadas en el sector urbano del Cantón Suscal de la provincia del Cañar- Ecuador. Es de metodologia descriptiva no experimental. Se afirma que ciertos docentes no contaban con debida capacitacion en las herramientas tecnologicas para enfrentar emergencia sanitaria de COVID-19, por cuanto no usaban frecuentemente la tecnologia de informacion y comunicación para impartir las clases en sus asisgnarutas que corresponden.

Descriptores: Tecnología de la información; aplicación informática; informática educativa; aprendizaje en línea. (Palabras tomadas de Tesauro UNESCO).

\begin{abstract}
The objective was to analyze the impact of COVID-19 in Basic and high school education: Urban educational units of Suscal - Ecuador. It was carried out in educational institutions located in the urban sector of Cantón Suscal in the province of Cañar-Ecuador. It is of descriptive non-experimental methodology. It is stated that certain teachers did not have adequate training in the technological tools to face the health emergency of COVID-19, since they did not frequently use information and communication technology to teach classes in their corresponding assistants.
\end{abstract}

Descriptors: Information technology; computer application; educational informatics; online learning. (Words taken from UNESCO Thesaurus). 


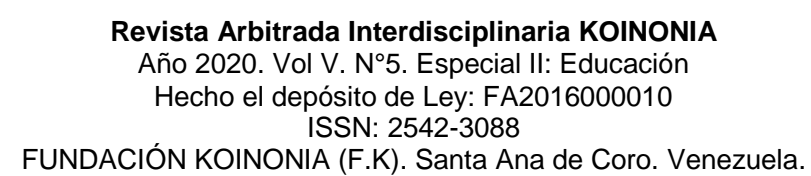

Luis Antonio Castro-Castro; Darwin Gabriel García-Herrera; Carlos Marcelo Ávila-Mediavilla; Juan Carlos Erazo-Álvarez

\section{INTRODUCCIÓN}

Las competencias digitales apuntan a la transformación en la educación. Por lo tanto, el uso de las tecnologías en la educación es muy importante en la actualidad y estar a la altura de la nueva era digital que está en la evolución diaria con el propósito de comprometer a una enseñanza de calidad, con el fin de que sea un óptimo aprendizaje para los estudiantes. Lamentablemente no era muy usado en procesos educativos en donde aplicaban procesos tradicionales ya acostumbrados a modalidad presencial durante mucho tiempo, resulta difícil acoger a las clases virtuales.

Cabe señalar que el rol docente en tiempos de COVID-19 ha cambiado a adaptación de modalidad virtual, (Oviedo, et al., 2014), mencionan que una de las características de los docentes es actualizarse en nuevos conocimientos tecnológicos, por cuanto la capacitación permanente nos llevara superar cualquier tipo de situación presentadas en este caso en sistema de educación.

Es interesante examinar el problema también lleva a los docentes a enfrentar nuevos retos y desafíos involucrando a todos los actores educativos en Suscal - Ecuador. Estamos en tiempos de cambios drásticos. Es así que la pedagogía tradicional se encuentra en jaque obligando adaptar a nuevas competencias para continuar formando a futuros profesionales.

Según la problemática se observa la suspensión de clases presenciales en las instituciones educativos tomando un rumbo distinto a lo tradicional como se acostumbraba, la cual permite entender que el rol docente juega un papel muy importante en la enseñanza sin importar que las clases impartidas sean presenciales o no presenciales.

De acuerdo con la publicación (EI Universo, 2020), el Ministerio de Gobierno y Educación suspendieron clases presenciales en todo el territorio ecuatoriano por declarar emergencia sanitaria desde el estado nacional. Donde se pronunció la ministra de Educación Monserrat Creamer para que los estudiantes no se atrasen pondrán a disposición una plataforma. 


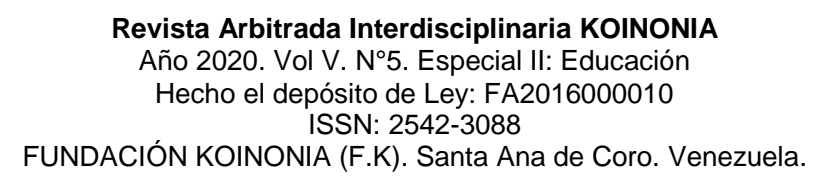

Luis Antonio Castro-Castro; Darwin Gabriel García-Herrera; Carlos Marcelo Ávila-Mediavilla; Juan Carlos Erazo-Álvarez

En cuanto a inicio de clases en régimen costa y galápagos 2020 - 2021 el Ministerio de Educación de Ecuador (Ministerio de Eeucación del Ecuador [MINEDU], 2020) se acata al cronograma escolar iniciando clases el 18 de mayo de 2020 en algunas instituciones y en otras 01 de junio de 2020 contando ya con Plan Educativo COVID-19 que habilitan a disposición recursos educativos, lineamientos y protocolos, guías para estudiantes y familias, información y recurso para docentes y textos escolares digitales.

Habría que decir también (Bonilla-Guachamín, 2020), afirma el cambio en educación ecuatoriana dando una solución a los cambios en la sociedad de conocimiento con nuevos retos tecnológicos. El uso de Tecnología de Información y Comunicación [TIC] implica selección de información y materiales según el contenido a abordar para que las clases no sean interrumpidas.

En este sentido, (Cáceres-Piñaloza, 2020), afirma que la interacción de los docentes y los estudiantes se optaran por medios virtuales, sacando el mayore de los provechos las aplicaciones digitales para video llamada tales como: WhatsApp, Facebook Live, Zoom siendo estos las más usadas particularmente de las otras, permitiendo a los estudiantes enriquecer los conocimientos de su alrededor, compartir ideas, proyectos, sugerencias desde su punto de vista y despejar dudas. Sea cierta o no esta acota que no todos disponen del Internet y los medios electrónicos.

Se tuvo como objetivo analizar el impacto de COVID-19 en Educación básica y bachillerato: Unidades educativas urbanas de Suscal - Ecuador.

\section{Referencial teórico}

Se puede mencionar algunos estudios ya realizados previos para la elaboración del presente proyecto con el fin de evidenciar el cambio que ha tenido la educación en tiempos de pandemia del COVID-19. enfocados al rol docente.

A continuación, (Tejada-Fernández \& Pozos-Pérez, 2018), en España manifiestan que es de suma importancia el desarrollo de las competencias tecnólogas en estos tiempos en sistema de educación. De mismo modo los docentes tienen que recibir capacitación 


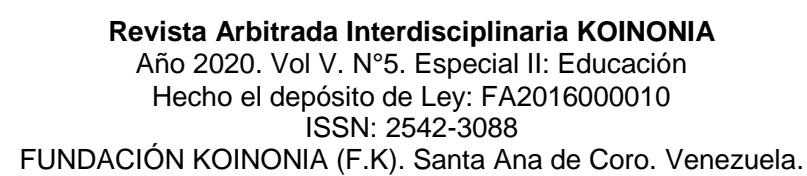

Luis Antonio Castro-Castro; Darwin Gabriel García-Herrera; Carlos Marcelo Ávila-Mediavilla; Juan Carlos Erazo-Álvarez

adecuada en el uso óptimo de las Tecnología de Información y Comunicación [TIC] para poner en práctica al momento de impartir clases de manera adecuada. No es cuestión de capacitar al docente más bien se requiere alfabetizar, aplicar e innovar. La nueva sociedad exige que sigan con los nuevos retos para orientar progresivamente al futuro con un desarrollo tecnológico del siglo XXI.

Por otra parte, (Toquero, 2020), en Filipinas manifiesta que por cumplir distanciamiento social se cerraron las puertas de las instituciones educativas, por tal sentido se vio afectada la educación superior por pandemia COVID-19, pero a la vez mejoró el modo educativo abriendo oportunidades al país enfocando a las tecnologías emergentes.

En este sentido, (Argandoña-Mendoza, et al., 2020), consideran un reto el tema de la investigación y educación en el Ecuador, durante el período de COVID.19, por cuanto se requiere forzosamente adaptar el proceso pedagógico tradicionalmente desarrollado en modalidad presencial a lo virtual, requiriéndose trabajar sobre la marcha en accionar un enfoque centrado en el estudiante, significando un reto para los actores educativos involucrados, por cuanto se requiere asumir, la educación multimedia, diseñar entornos virtuales de aprendizajes (EVA), en razón de proyectar una didáctica armonizada con el logro de una educación transformadora. En otras instituciones ya fueron implementados desde algún tiempo atrás, es un apoyo para a las exigencias del sistema de educación en estos tiempos. Es una estrategia para llegar a un aprendizaje significativo orientados a los objetivos planteados, por cuanto la sociedad actual obliga a estar a la vanguardia de la misma.

Y, además: el (Chancusig-Chisag, et al., 2017), en el mismo contexto manifiestan que la educación del siglo XXI la Tecnología de Información y Comunicación [TIC] son sumamente importantes en el proceso de enseñanza por la razón debe continuar con la capacitación para seguir involucrado en el mundo de la tecnología y a la vez formando profesionalmente. La mayoría de los docentes tienen claro el concepto de herramientas digitales, pero no todos ponen en práctica ya sea por tiempo o carencia de dominio en la tecnología, también consideradas como un apoyo alternativo para la enseñanza de este 
Luis Antonio Castro-Castro; Darwin Gabriel García-Herrera; Carlos Marcelo Ávila-Mediavilla; Juan Carlos Erazo-Álvarez

modo siguen quedando en el proceso tradicional, aislados de los avances tecnológicos que hoy el sistema de educación exige.

Desde el punto de vista de (Livari, et al., 2020), la pandemia de COVID-19 en la educación básica nos obliga a dar un salto digital, las instituciones educativas enfrentaron la transformación digital al contrario los niños y jóvenes no todos cuenta con uso y el acceso a de la tecnología, así como la competencia para la dicha transformación. También se debe considerar cómo podríamos capacitar a los docentes. El sistema de educación es una más de las víctimas de pandemia COVID-19, ante esta situación la educación debe continuar llevados a giros estratégicos para continuar con las clases.

Basándose en (Rey \& Jabonero, 2018), consideran la existencia de quienes no conocen a fondo el sistema educativo actual por cuanto piensan en la presencia de analfabetismo sin tomar en cuenta que ellos estudiaron en tiempos anteriores y el acceso a los estudios fueron muy pocos. El profesor debe enseñar contenidos con un modelo pedagógico más actualizado involucrando el uso de la tecnología, en donde la enseñanza apunte a inteligencia emocional trasmitiendo valores mediante la aplicación de la Tecnología de la Información y Comunicación [TIC] y de la Tecnología para el Aprendizaje y Conocimiento [TAC] de echo las planificaciones de clases toman otros procedimientos basando en proceso de educación digital.

Desde luego según (Parra \& Keyla, 2014), sostienen que la práctica de intercambio cooperativo entre estudiante docente permite un aprendizaje significativo en el proceso de enseñanza aprendizaje. La interacción entre docente y estudiante debe practicar dinamismo y el momento de clase siempre debe ser diferente, ya que el estudiante está en constante cambio de conocimiento y experiencia. De tal modo se practica involucrar tanto estudiante, profesor, padre de familia y directivo. Siendo el aprendizaje la responsabilidad del estudiante.

Como afirma (Vargas Martínez, 2020), que está expuesto a cambios el rol docente en los últimos tiempos y es más notable, por cuanto se desvanece el confort en la labor docente siendo habitual el uso de pizarra y las guías. Al ser parte de la era digital son los docentes 


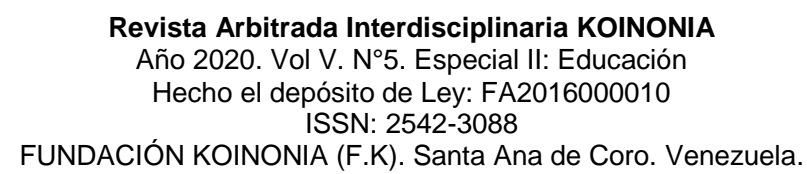

Luis Antonio Castro-Castro; Darwin Gabriel García-Herrera; Carlos Marcelo Ávila-Mediavilla; Juan Carlos Erazo-Álvarez

quienes están inmersos a migrar, formar, actualizar para promover clases acordes a la actualidad.

Además, (Villafuerte Holguín, et al., 2020), mencionan que las autoridades de cada institución educativa tienen la obligación de capacitar en el uso de las diferentes aplicaciones tecnológicas ya que la educación virtual no es solo cargar y descargar archivos multimedia y texto. Para llevar una clase de ambiente confort debe aplicar las mejores estrategias sacando provecho de las herramientas digitales. Se deberá enfrentar la pandemia COVID-19 aplicando mejores estrategias donde las clases sincrónicas tengan siguientes momentos:

1. Inicio: donde se involucra el saludo, motivación y objetivos de clases.

2. Desarrollo: referirse al tema del objetivo mediante foros, presentaciones y otros y al finalizar respuestas de dudas de la misma.

3. Cierre: evaluación de la teoría abordada y despedida con mensaje motivadora.

\section{METODOLOGÍA}

La presente investigación fue realizada en las instituciones educativas ubicadas en el sector urbano del Cantón Suscal de la provincia del Cañar- Ecuador. Es de metodologia descriptiva no experimental, se tomó como población, docentes de los niveles de educación básica y bachillerato, la muestra estuvo compuesta por 10 profesores, la misma se puede ver en la tabla 1.

Se empleó como técnica de recolección de información, la encuesta, como instrumento un cuestionario en escala de tipo Likert con una valoracion de poco (1) y Mucho (5), correspondiendose a 7 items. Validandose el cuestionario en función de juicio de expertos y calculo de fiabilidad de Alfa de Cronbach dando como resultado 0,783. Los datos recopilados, fueron procesados en razón de la estadistica descriptiva y chi cuadrado de Pearson, con apoyo del programa IBM SPSS Stadistics V19. 


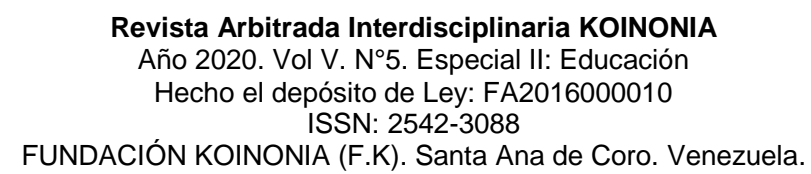

Luis Antonio Castro-Castro; Darwin Gabriel García-Herrera; Carlos Marcelo Ávila-Mediavilla; Juan Carlos Erazo-Álvarez

\section{Tabla 1}

Descripcion de los participantes.

\begin{tabular}{cccc}
\hline Encuesta & Genero & Lugar de Trabajo & Tiempo Docente \\
\hline E1 & Masculino & Suscal & $11-$ mas años \\
E2 & Masculino & Suscal & $6-10$ años \\
E3 & Femenino & Suscal & $1-5$ años \\
E4 & Femenino & Suscal & $1-5$ años \\
E5 & Femenino & Suscal & $6-10$ años \\
E6 & Femenino & Suscal & $11-$ mas años \\
E7 & Masculino & Suscal & $6-10$ años \\
E8 & Femenino & Suscal & $1-5$ años \\
E9 & Masculino & Suscal & $1-5$ años \\
E10 & Masculino & Suscal & $6-10$ años \\
\hline
\end{tabular}

Fuente: Elaboracion propia.

\section{RESULTADOS}

Por pandemia de COVID-19, se ha acogido a teletrabajo que esta desarrollando por difrentes opciones.

\section{Tabla 2}

Estadísticos descriptivos sobre frecuencia de uso de medios digitales antes de pandemia COVID1-9 y se preparó para impartir clases durante el tiempo de pandemia COVID-19.

\begin{tabular}{lcc}
\hline & $\mathrm{N}$ & Media \\
\hline En su asignatura hacia uso de los medios digitales antes de & 10 & 2,70 \\
COVID-19 para impartir clases. & 10 & 3,30 \\
$\begin{array}{l}\text { Se ha preparado en el uso de TICS para llevar a cabo el proceso } \\
\text { de enseñanza durante el tiempo de COVID-19. }\end{array}$ & 10
\end{tabular}

Fuente: Elaboracion propia. 
Luis Antonio Castro-Castro; Darwin Gabriel García-Herrera; Carlos Marcelo Ávila-Mediavilla; Juan Carlos Erazo-Álvarez

Se puede evidenciar que el resultado obtenido del interrogante la media es 2.70 en donde se envidencia que los docentes en su asignatura hacían poco uso de los medios digitales antes de COVID-19 para impartir clases, del mismo modo en el siguiente interrogante da una media de 3.30, en donde se evidencia claramente que se han preparado en el uso de TICS para llevar a cabo el proceso de enseñanza durante el tiempo de COVID-19 pero no es suficiente según él resultado.

\section{Tabla 3 .}

Estadísticos descriptivos para obtener un grado de satisfecho en el nivel de uso de la tecnología para llegar a los objetivos de su asignatura y se sienten satisfecho enfrentando nuevos desafíos en tiempo de COVID-19.

\begin{tabular}{lcc}
\hline & $\mathrm{N}$ & Media \\
\hline $\begin{array}{l}\text { Se siente satisfecho usando la tecnología para llegar a los objetivos } \\
\text { de su asignatura. }\end{array}$ & 10 & 3,40 \\
Se siente satisfecho enfrentando nuevos desafíos en tiempo de & 10 & 3,30 \\
COVID-19. & 10 \\
N válido (según lista) & 10 \\
\hline
\end{tabular}

Fuente: Elaboracion propia.

Se puede llegar al análisis en el interrogante de acuerdo al resultado de media 3.40 que no están tan satisfecho usando la tecnología para llegar a los objetivos de su asignatura y según el valor de la media 3.30 del interrogante tampoco están muy satisfechos enfrentando nuevos desafíos en tiempo de COVID-19. 
Revista Arbitrada Interdisciplinaria KOINONIA

Año 2020. Vol V. N5. Especial II: Educación

Hecho el depósito de Ley: FA2016000010

ISSN: 2542-3088

FUNDACIÓN KOINONIA (F.K). Santa Ana de Coro. Venezuela.

Luis Antonio Castro-Castro; Darwin Gabriel García-Herrera; Carlos Marcelo Ávila-Mediavilla; Juan Carlos Erazo-Álvarez

\section{Tabla 4.}

Estadísticos descriptivos para ver si están muy de acuerdo con siguientes interrogantes.

\begin{tabular}{lcc}
\hline & $\mathrm{N}$ & Media \\
\hline Está de acuerdo que el rol docente ha cambiado en este tiempo de COVID-19. & 10 & 4,70 \\
Está de acuerdo que sean capacitados frecuentemente en el uso de herramientas & 10 & 4,90 \\
digitales en tiempo de COVID-19 & & \\
Está de acuerdo que la educación básica y bachillerato usen medios digitales en el & 10 & 4,60 \\
proceso de aprendizaje en tiempo de COVID-19 & 10 \\
N válido (según lista) & 10 \\
\hline
\end{tabular}

\section{Fuente: Elaboracion propia}

Se valora el resultado en la interrogante dando una media de 4.70, en donde se puede apreciar que están de acuerdo que el rol docente ha cambiado en este tiempo de COVID-19, de mismo modo en siguiente interrogante se evidencia el valor de media 4.90, que están más que de acuerdo que sean capacitados frecuentemente en el uso de herramientas digitales en tiempo de COVID-19 y en ultimo interrogante arroja el resultado de la moda 4.60, en donde se puede interpretar que están Está de acuerdo que la educación básica y bachillerato usen medios digitales en el proceso de aprendizaje en tiempo de COVID-19 
Luis Antonio Castro-Castro; Darwin Gabriel García-Herrera; Carlos Marcelo Ávila-Mediavilla; Juan Carlos Erazo-Álvarez

\section{PROPUESTA}

Según los resultados en mención, se propone una capacitación a los docentes en uso de las herramientas tecnológicas innovadoras para mejorar la enseñanza en las instituciones educativas ubicadas en el sector urbano del Cantón Suscal de la provincia del Cañar.
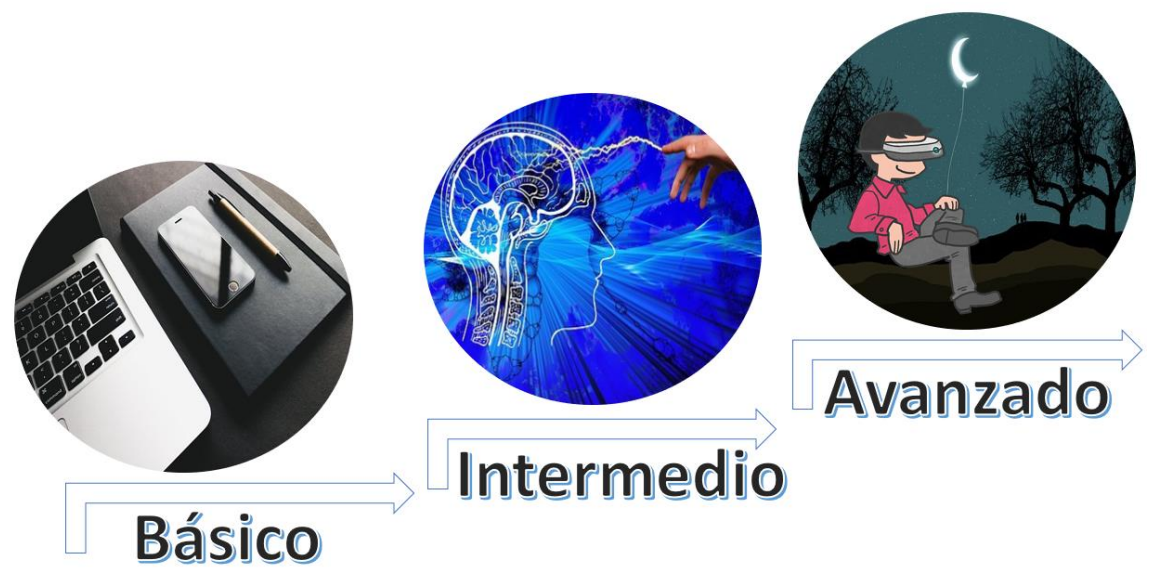

Figura 1. Niveles de capacitación.

Fuente: Elaboración propia.

Para la propuesta de la presente capacitación se considera emplear metodología de carácter descriptivo, centrado en la práctica docente con la finalidad de brindar una buena calidad de educación el proceso enseñanza aprendizaje a través del mundo virtual.

La capacitación propuesta cuenta con tres niveles donde se puede observar en Figura 1. Nivel básico. - para docentes nuevos o los que nunca han utilizado herramientas tecnológicas. Nivel intermedio. - para docentes que conocen las herramientas tecnológicas de presentación del siglo XXI. Nivel avanzado. - para aquellos docentes que cuentan con experiencia considerable con capacitación previa y deseen convertirse en expertos.

Cabe recalcar que no será ninguna obligación de asistir a los tres niveles si en caso de que ya tiene una capacitación previa a continuación se detalla los contenidos en cada uno de los niveles. 
Luis Antonio Castro-Castro; Darwin Gabriel García-Herrera; Carlos Marcelo Ávila-Mediavilla; Juan Carlos Erazo-Álvarez

Nivel básico. - Este nivel está dedicado a docentes nuevos o los que no han utilizado herramientas digitales en el proceso de enseñanza. Proporciona una instrucción a la utilización de herramientas de presentación para crear presentaciones interactivas, Mapas Mentales, Infografía, logo, contar historietas entre otros. Todo esto empleando los recursos didácticos del Siglo XXI. Proporcionando una amplia instrucción en los componentes, características y sus funciones, así como también en la utilización adecuando.

Presentando la información de las clases para llegar al objetivo de la materia con color y movimiento. Personalizando el contenido de manera genial y única. Siempre causando interacción y movilización al estudiante.

La capacitación se compone de tres herramientas tecnológicas en este nivel. Tales como: Genially, Canva, Inklewriter

Nivel intermedio. - Los docentes que han concluido el nivel básico están listos para avanzar hacia la capacitación de "nivel intermedio". En el presente nivel la capacitación proporciona una instrucción en herramientas para diagnósticos y evoluciones interactivas. Desarrolla el conocimiento y estrategias a la hora de evaluar, ya que es el momento de aplicar de modo correcto y adecuado las herramientas tecnológicas sin poner en tensión a los estudiantes, usando la creatividad, imaginación y un buen uso de las mismas.

Se emplea las herramientas que sean accesibles y portables. Tales características que diferencia con los demás. Otros temas clave son la optimización y la eficiencia de a la hora de emplear, y la importancia de las herramientas de evaluación integrado en las asignaturas que imparte cada uno de los docentes de nivel básico y bachillerato.

La dicha capacitación en las Herramientas de Evaluación Interactiva en el nivel ya antes en mención es: Kahoot, Padlet, Lesoon Plans, Quizizz

Nivel avanzado. - La capacitación en el presente nivel se imparte para aquellos docentes experimentados, con conocimiento considerable y capacitación previa y que estén orientados a convertirse en expertos. Se instruirá sobre mundos virtuales y realidad 


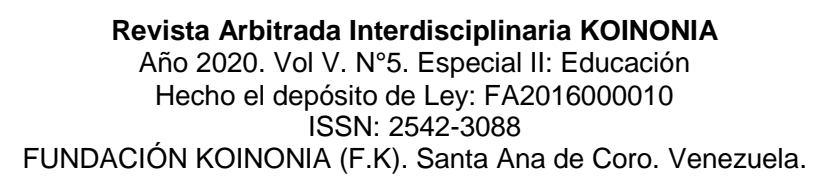

Luis Antonio Castro-Castro; Darwin Gabriel García-Herrera; Carlos Marcelo Ávila-Mediavilla; Juan Carlos Erazo-Álvarez

aumentada, donde la tecnología y la magia se apodera del aula virtual. Aquí se ofrece una serie de programas que promoverán la curiosidad, la magia y la adrenalina en los estudiantes. Se debe tener presente que un docente es el diseñador del ambiente digital para ofrecer un contenido real sobre cada materia que se imparte.

Se instruirá en la característica principal que es la inmersión en el mundo virtual a través de la simulación de un ambiente real.

El presente nivel engloba instrucción en siguientes herramientas digitales: Sploder, Roblox, Metaverse

\section{CONCLUSIONES}

Se afirma que ciertos docentes no contaban con debida capacitacion en las herramientas tecnologicas para enfrentar emergencia sanitaria de COVID-19, ya que no usaban frecuentemente la tecnologia de informacion y comunicación para impartir las clases en sus asisgnarutas que corresponden.

En estos tiempos que el mundo esta atravesando se covierte en uno de los desafios primoridales que requieren ser capacitados en las herrramientas tecnolocias, con el unico proposito de brindar una educacion de calidad tanto a los niveles basico y bachillerato en las unidades educativas rurales de canton Suscal.

Se hanela la transformacion de sistema de educacion que integren en las asignaturas de educación básica y bachillerato medios digitales en el proceso de enseñanza aprendizaje en tiempo de COVID-19 y a futuro. De esta manera llegaran a una satisfaccion usando la tecnología para llegar a los objetivos de sus asignaturas.

\section{FINANCIAMIENTO}

No monetario 


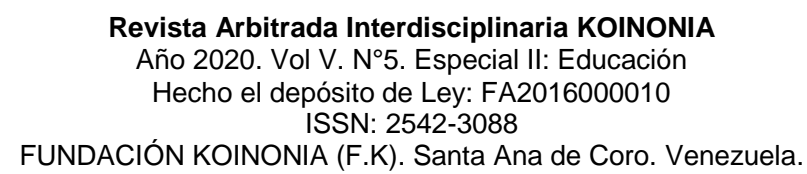

Luis Antonio Castro-Castro; Darwin Gabriel García-Herrera; Carlos Marcelo Ávila-Mediavilla; Juan Carlos Erazo-Álvarez

\section{AGRADECIMIENTO}

A las instituciones educativas ubicadas en el sector urbano del Cantón Suscal de la provincia del Cañar- Ecuador; por apoyar el desarrollo de la investigación.

\section{REFERENCIAS CONSULTADAS}

Argandoña-Mendoza, M., García-Mejía, R., Ayón-Parrales, E., \& Zambrano-Zambrano, Y. (2020). Investigación e innovación educativa: Reto escolar por COVID-19 en el Ecuador. [Educational research and innovation: School challenge due to COVID19 in Ecuador]. EPISTEME KOINONIA, 3(5), 162-182. http://dx.doi.org/10.35381/e.k.v3i5.726

Bonilla-Guachamín, J. A. (2020). Las dos caras de la educación en el COVID-19. [The two faces of education in COVID-19]. CienciAméRica, 9(2), 89-98. http://dx.doi.org/10.33210/ca.v9i2.294

Cáceres-Piñaloza, K. F. (2020). Convivencia y aprendizaje en tiempos de COVID-19. [Coexistence and learning in times of COVID-19]. CienciAméRica, 9(2), 38-44. http://dx.doi.org/10.33210/ca.v9i2.284

Chancusig-Chisag, J. C., Flores-Lagla, G. A., \& Constante, M. F. (2017). Las TIC'S en la formación de los docentes. [ICTs in teacher training]. Revista Boletín Redipe, 6(2), 174-198.

El Universo. (2020). Coronavirus: Se suspenden clases en Ecuador, no se define fecha de retorno. [Coronavirus: Classes are suspended in Ecuador, return date is not defined]. Recuperado de https://n9.cl/pzb2

Livari, N., Sharma, S., \& Ventä-Olkkonen, L. (2020). Digital transformation of everyday life-How COVID-19 pandemictransformed the basic education of the young generation and whyinformation management research should care? International Journal of Information Management, 55; 1-6. https://doi.org/10.1016/j.ijinfomgt.2020.102183 
Revista Arbitrada Interdisciplinaria KOINONIA

Año 2020. Vol V. N5. Especial II: Educación

Hecho el depósito de Ley: FA2016000010

ISSN: 2542-3088

FUNDACIÓN KOINONIA (F.K). Santa Ana de Coro. Venezuela.

Luis Antonio Castro-Castro; Darwin Gabriel García-Herrera; Carlos Marcelo Ávila-Mediavilla; Juan Carlos Erazo-Álvarez

Ministerio de Educación del Ecuador [MINEDU]. (2020). Cronograma escolar régimen Costa y Galápagos año lectivo 2020-2021. [School schedule for the Costa and Galapagos regime school year 2020-2021]. Recuperado de https://n9.cl/hqx7

Oviedo, P. E., \& Pastrana Armírola, L. H. (2014). Investigaciones y desafíos para la docencia del siglo XXI Titulo. [Research and challenges for teaching in the XXI century Title]. Bogotá D.C., Colombia: Kimpres. Obtenido de https://n9.cl/0ut6

Parra, F., \& Keyla, N. (2014). El docente y el uso de la mediación en los procesos de enseñanza y aprendizaje. [The teacher and the use of mediation in the teaching and learning processes]. Revista de Investigación, 38(83),155-180

Rey, F., \& Jabonero, M. (2018). Sistemas educativos docentes [Teacher education systems]. Fundación Santillana. Recuperado de https://n9.cl/bojvy

Tejada-Fernández, J., \& Pozos-Pérez, K. (2018). Nuevos escenarios y competencias digitales docentes: hacia la profecionalización docente con TIC. [New scenarios and digital teaching skills: towards the professionalization of teachers with ICT]. Profesorado, 22(1); 25 - 51.

Toquero, C. M. (2020). Challenges and Opportunities for Higher Education amid the COVID-19 Pandemic: The Philippine Context. Pedagogical Research, 5(4), em0063. https://doi.org/10.29333/pr/7947

Vargas-Martínez, D. F. (2020). La enseñanza del Covid-19 con relación a la educación 3.0. [The teaching of Covid-19 in relation to education 3.0]. Revista Ideales, 11(1); 85-89.

Villafuerte-Holguín, J. S., Bello Piguave, J. E., Pantaleón Cevallos , Y., \& Bermello Vidal, J. O. (2020). Rol de los docentes ante la crisis del COVID-19, una mirada desde el enfoque humano. [Teachers' role in the COVID-19 crisis, a human perspective]. Revista Electrónica Formación y Calidad Educativa, 8(1); 132-150.

C2020 por los autores. Este artículo es de acceso abierto y distribuido según los términos y condiciones de la licencia Creative Commons Atribución-NoComercial-Compartirlgual 4.0 Internacional (CC BY-NC-SA 4.0)

(https://creativecommons.org/licenses/by-nc-sa/4.0/). 